\title{
Generalized Purpuric Lichen Nitidus in a 32-Year-Old Filipino Male with Acute Myeloid Leukemia: Case Report
}

\author{
Soraya Elisse E. Escandor, MD, Nicole Marella G. Tan, MD, \\ Val Constantine S. Cua, MD and Eileen Leisl A. Cubillan, MD \\ Department of Dermatology, Philippine General Hospital, University of the Philippines Manila
}

\begin{abstract}
A 32-year-old Filipino male managed as a case of acute myeloid leukemia presented with a 6-month history of generalized, red-brown papules. After chemotherapy, the patient was advised 30-minutes daily sun exposure as a form of natural phototherapy. On follow-up, most lesions flattened. This case demonstrates chemotherapy's potential role in controlling the patient's inflammatory state and using ultraviolet light therapy in the form of sunlight to manage generalized purpuric lichen nitidus.
\end{abstract}

Keywords: Lichen Nitidus, Purpuric Lichen Nitidus, Dermoscopy, Phototherapy

\section{INTRODUCTION}

Lichen nitidus is an idiopathic lichenoid eruption described by Felix Pinkus in $1907 .{ }^{1}$ It is characterized by multiple $1-2 \mathrm{~mm}$ discrete smooth, round domeshaped papules that are often localized on the trunk, face, extremities, and genitalia. It is usually asymptomatic but may be pruritic in some cases. ${ }^{2}$ Generalized purpuric lichen nitidus is rare, exhibiting similar lesions to the classic variant but with red-brown papules, which correspond to the presence of siderophages in the dermis. We herein report a rare case of generalized purpuric lichen nitidus with characteristic dermoscopic findings.

\section{CASE REPORT}

A 32-year-old Filipino man presented with diffuse mildly pruritic $1-2 \mathrm{~mm}$ red-brown discrete monomorphic papules covering the face, trunk, and extremities. He had a six-month history of multiple grouped red-brown nonpruritic papules, which initially appeared on the left lower leg. This later progressed to involve the rest of the body in a caudocephalad direction. Self-medication with sulfur soap did not lead to improvement of lesions. Two months later, the patient consulted at a local clinic where a punch biopsy revealed lichenoid dermatitis. On laboratory examination, he had anemia and thrombocytopenia. He was referred to our institution for further workup, where his bone marrow biopsy result was consistent with Acute Myeloid Leukemia. Before the present illness, the patient had no known dermatologic conditions and an unremarkable personal 
and family medical history. He is an 18-pack-a-year smoker, a regular alcoholic consumer. He denies illicit drug use.

Physical examination revealed multiple, well-demarcated, red-brown, firm, shiny monomorphic papules distributed on the face, scalp, neck, trunk, genitalia, buttocks, and the upper and lower extremities (Figure 1A-G). These did not blanch on diascopy. No nail and mucosal involvement were seen.

\section{Dermatoscopic findings}

Dermoscopy of the lesions on the back revealed multiple well-demarcated concentric regions characterized by a structureless ill-defined whitish annular cloud-like area and a peripheral halo with erythematous-brown pigmentation (Figure 2A).

On the right leg, red globules and dots overlying faintly red homogeneous areas were prominent (Figure 2B).
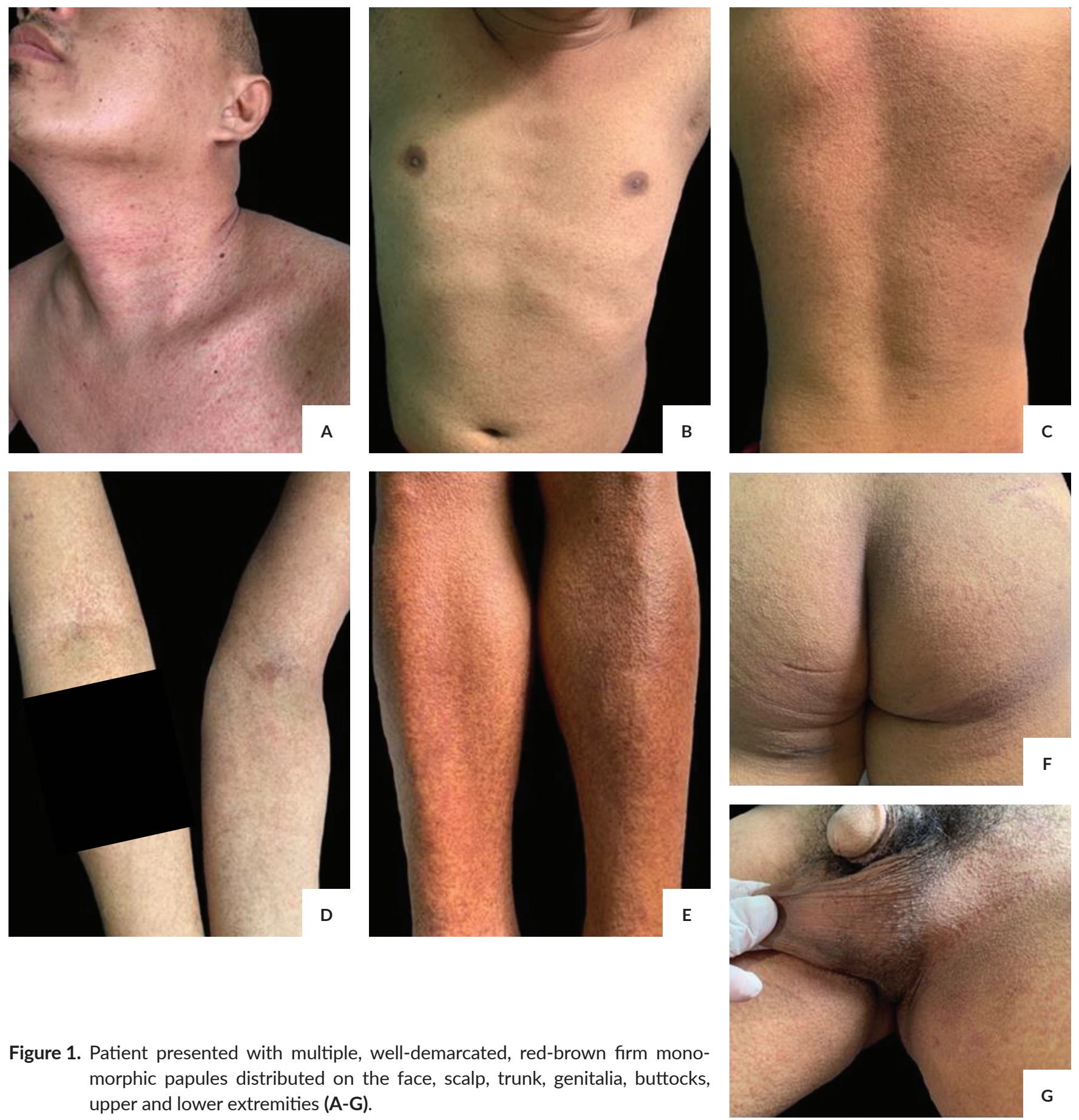

Figure 1. Patient presented with multiple, well-demarcated, red-brown firm monomorphic papules distributed on the face, scalp, trunk, genitalia, buttocks, upper and lower extremities (A-G). 


\section{Histopathologic findings}

Biopsies were done on the back, and right lower leg; both showed findings of patchy lichenoid infiltrates of lymphocytes and histiocytes in the dermis. The specimen from the back was more characteristic for lichen nitidus, with the infiltrates spanning 3-4 rete ridges in the papillary dermis, resembling a "claw clutching a ball." 3 Moreover, extravasated erythrocytes and siderophages were appreciated within the infiltrates. The overlying epidermis showed basketweave orthokeratosis, parakeratosis, and focal vacuolar interface changes (Figure 3A). Perls Prussian blue staining revealed numerous siderophages in the dermis (Figure 3B).
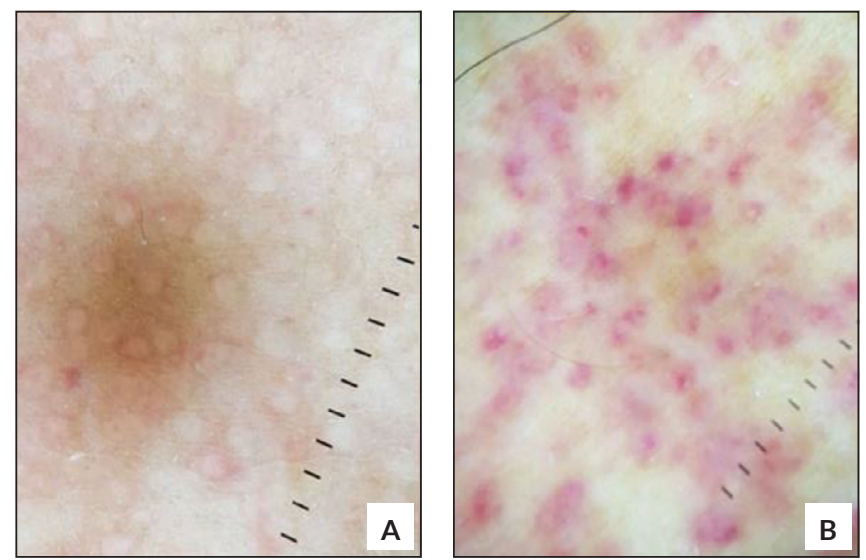

Figure 2. Dermatoscopic findings demonstrating multiple concentric regions with whitish annular cloud-like area, and a peripheral halo with erythematousbrown pigmentation (A). Red globules and red dots overlying faintly red homogeneous areas were more prominent in the leg (B).
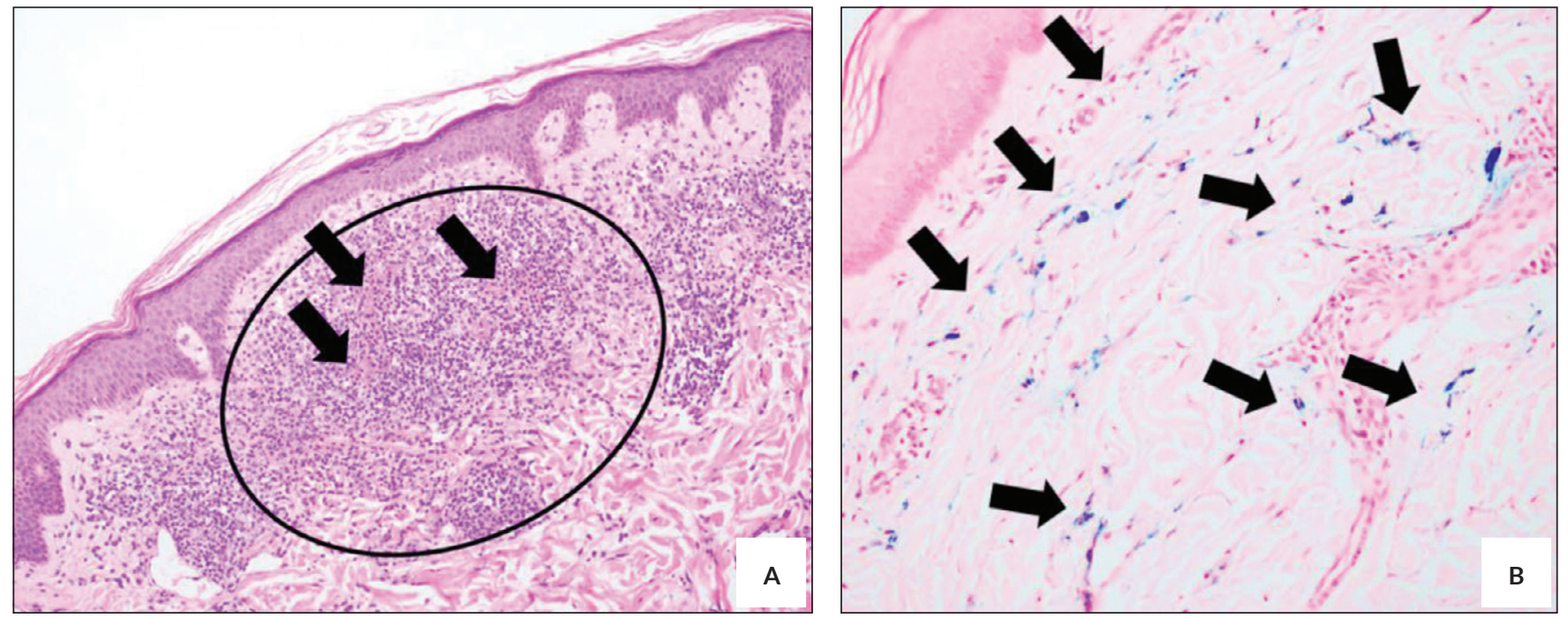

Figure 3. Biopsy of the back and right lower leg. (A, B). There is basketweave orthokeratosis, patchy lichenoid infiltrates (black circle) of lymphocytes and histiocytes spanning 3-4 rete ridges in the papillary to superficial dermis, with overlying parakeratosis and focal vacuolar interface changes. Perls Prussian blue stain revealed hemosiderin deposits in the dermis (B). 
and morphology and their histopathologic findings of lymphohistiocytic infiltrate. ${ }^{6}$ Though these two conditions may co-exist, several cases have shown that distinct features can differentiate the two. Clinically, lichen nitidus is described as discrete, monomorphous, papular lesions, while lichen planus presents purple, polygonal papules and plaques with white scale. Histopathologically, lichen nitidus demonstrates heterogeneous infiltration, while lichen planus has a more uniform presentation for its lymphocytic infiltrate, mostly CD4+ T helper cells. ${ }^{7}$

Lichen nitidus has a diverse clinical presentation. Apart from being either localized or generalized, other described variants include purpuric, actinic, follicular, linear, and vesicular types. ${ }^{8}$ Each of these is considered to be quite rare. Purpuric lichen nitidus presents similarly to the classic variant but appears red-brown. ${ }^{5}$
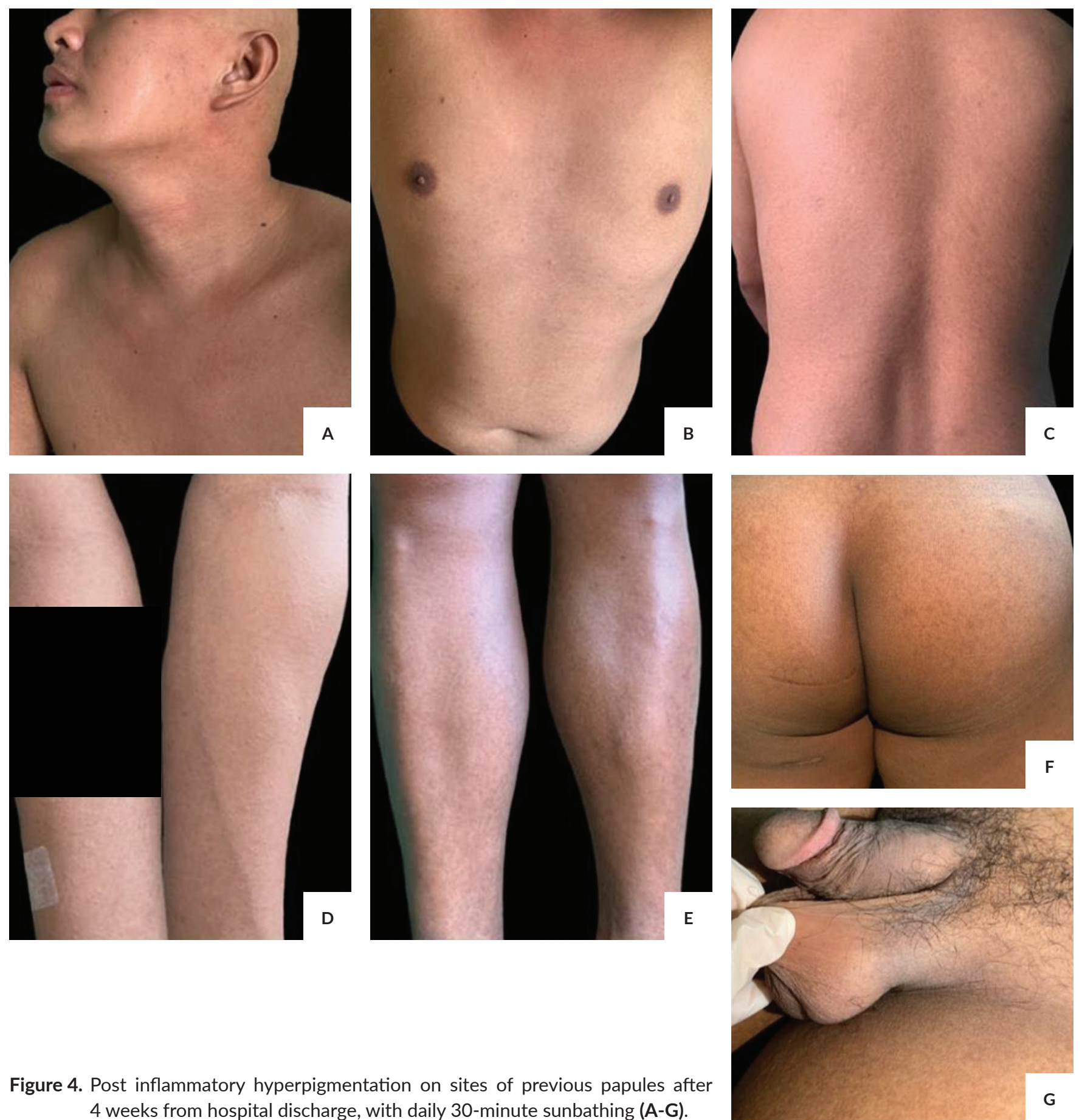

Figure 4. Post inflammatory hyperpigmentation on sites of previous papules after 4 weeks from hospital discharge, with daily 30 -minute sunbathing (A-G).

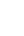


There are only three published cases of generalized purpuric lichen nitidus, all of which describe lesions developing initially on the lower legs, similar to what was observed in our patient's case. Also, similarly, these papules gradually increased in number, eventually becoming generalized. ${ }^{9}$

The dermoscopic findings of lichen nitidus include the absence of dermatoglyphics, radial ridges, and central circular depression under nonpolarized light. Using polarized light dermoscopy, ill-defined whitish areas with erythema, vessels, and peripheral scaling are seen. A faint brown shadow within these whitish areas may also be seen. The absence of dermatoglyphics correlates with the thin overlying epidermis over the infiltrates. The whitish areas possibly correspond to epidermal acanthosis. ${ }^{3}$ The central depression and illdistinct brown shadow correlate with the lymphohistiocytic infiltration while the radial ridges correspond to the elongation of the rete ridges. Scaling may correlate with parakeratosis seen histopathologically in the lesion. ${ }^{10}$ Purpuric lichen nitidus may have an additional feature of red globules and red dots overlying faintly red homogeneous areas, corresponding to extravasated erythrocytes. ${ }^{10}$ Clinical differential diagnoses include lichen planus, lichen scrofulosorum, keratosis pilaris rubra, pityriasis rubra pilaris, frictional lichenoid dermatitis, and papular urticaria. ${ }^{11}$

The histopathologic findings of variants such as generalized purpuric lichen nitidus share the characteristic hallmarks of the classic disease. This is often described as a ball-in-claw configuration, seen as dense dermal lymphohistiocytic infiltrate, encompassed by elongated rete ridges. ${ }^{2}$ Findings such as infiltrates of neutrophils, eosinophils, and plasma cells are rare but may still be seen in lichenoid tissue reactions. ${ }^{8,12}$ Additional findings of hyalinized vessels and sub-epidermal extravasation of erythrocytes may be seen in purpuric lichen nitidus. In our patient, such changes were attributed to thrombocytopenia, a common finding in acute myeloid leukemia. ${ }^{13}$

Lichen nitidus has an unpredictable clinical course and usually resolves spontaneously after a few months to years from the onset in the majority of patients. Post-inflammatory hyperpigmented macules often develop as the papules resolve. Treatment usually is reserved for symptomatic or generalized patients. ${ }^{3}$

There is no current standard of care for generalized lichen nitidus, but several case reports have been published to show possible treatment modalities and effects. A case of an asymptomatic generalized lichen nitidus in a 10 -yearold male patient was observed and not given any treatment. The lesions cleared spontaneously within 6-8 months. ${ }^{14}$ Another asymptomatic case in a 7-year-old male with neuro-fibromatosis type 1 and juvenile myelomonocytic leukemia was published. He presented with a one-month history of generalized lichen nitidus and was treated with emollients. ${ }^{15}$ Five more cases with varying ages and sex were treated with corticosteroids. Varied results were noted, with two having a resolution of lesions and one with the persistence of lesions. ${ }^{7,16-18}$

There are promising results on the use of sunlight and ultraviolet light, with mild to complete resolution in some and striking improvement of lesions on photo-exposed skin areas in others. One such case is a 32-year-old male with a 1-year history of generalized lichen nitidus treated with topical clobetasol propionate $0.05 \%$ ointment and triamcinolone acetonide $0.1 \%$ cream. The authors noted significant resolution of lesions in photo-exposed areas and suggested that ultraviolet light treatment could have played a role. ${ }^{2}$ In 1986 , a case report described a 27 -year-old female with generalized lichen nitidus that had previously been unsuccessfully treated with corticosteroids, who then improved after nine weeks of PUVA. ${ }^{19}$ Meanwhile, total clearing was observed in an 80-year-old female after being managed initially with UVA/UVB and astemizole for two weeks and later with light treatment and prednisolone for another four weeks. A 19-year-old female with a two-year history of generalized follicular spinous lichen nitidus was treated with oral PUVASoL and topical corticosteroids with mild improvement. She was later managed with oral prednisolone and dapsone, which resulted in significant clearance of skin lesions. ${ }^{20}$

The use of natural sunlight therapy for lichen nitidus was reported in a six-year-old male patient by Berman, Truong, and Cheng in 2019. He was previously managed with topical corticosteroids for several months without any improvement. NB-UVB phototherapy was initially considered, but it was deemed impractical due to the distance of the patient's house to the clinic. Hence, he was managed with daily natural sunlight therapy of at least 15 minutes in duration, with concomitant topical corticosteroids. Three months later, the patient had complete resolution of the eruption. ${ }^{21}$

Apart from sunlight therapy, our patient was also treated for acute myeloid leukemia. Treatment of established cutaneous manifestations of acute myeloid leukemia such as leukemia cutis, paraneoplastic dermatosis, and cytopeniarelated dermatosis is directed at their underlying etiology. Chemotherapy and supportive agents are employed. ${ }^{22} \mathrm{~A}$ review on case reports of cutaneous presentations in acute leukemia, with leukemia cutis appearing in as much as $20 \%$ of patients, was published in 2017. Three cases of a 67-yearold female, a 68-year-old male, and a 67-year-old male had remission after chemotherapy. ${ }^{23}$

Acute myeloid leukemia is a disease state with a dysregulated immune system and presents with bone marrow failure features-including cytopenias that can manifest as purpura. ${ }^{13}$ Meanwhile, lichen nitidus is described as an inflammatory eruption. While there is no published literature on the relationship between these two diseases, this case report featured a patient's lesions that evolved 
when he was diagnosed with leukemia and resolved four weeks post-induction chemotherapy. Hence a possible association between the two cannot be disregarded.

\section{CONCLUSION}

Lichen nitidus is a lymphocytic cutaneous eruption with an unknown etiology, making it difficult to institute targeted therapy. We report a case of generalized purpuric lichen nitidus, a rare variant. The clinical feature of redbrown papules and its dermoscopic and histopathologic findings distinguishes it from the classic variant.

While most cases of lichen nitidus resolve spontaneously, this case supports previous reports which reinforce the role of ultraviolet light therapy and natural sunlight therapy as valuable tools in managing generalized lichen nitidus. Moreover, purpuric lichen nitidus as a paraneoplastic manifestation of acute leukemia may also be considered.

\section{Statement of Authorship}

All authors participated in the data collection and analysis and approved the final version submitted.

\section{Author Disclosure}

All authors declared no conflicts of interest.

\section{Funding Source}

This study has no funding support.

\section{REFERENCES}

1. Cheng C-Y, Chiu C-S, Huang Y-H. Palmar Purpuric Lichen NitidusClinicopathological and Dermoscopic Findings. Dermatologica Sinica. 2012Sep12;31(3):137-9.

2. Arizaga AT, Gaughan MD, Bang RH. Generalized Lichen Nitidus. Clin Exp Dermatol. 2002 Mar;27(2):115-7. doi: 10.1046/j.13652230.2002.00971.x. PMID: 11952701.

3. Chu J, Lam JM. Lichen Nitidus. CMAJ. 2014 Dec 9;186(18):E688. doi: 10.1503/cmaj.140434. Epub 2014 Oct 27. PMID: 25349001; PMCID: PMC4259797.

4. Pinkus H, Shair HM. Koebner Phenomenon in Lichen Nitidus; Report of Two Cases. AMA Arch Derm Syphilol. 1952 Jan;65(1):827. doi: 10.1001/archderm.1952.01530200086012. PMID: 14877293.

5. Yáñez S, Val-Bernal JF. Purpuric Generalized Lichen Nitidus: An Unusual Eruption Simulating Pigmented Purpuric Dermatosis. Dermatology. 2004;208(2):167-70. doi: 10.1159/000076495. PMID: 15057011.

6. Ellis FA. Is Lichen Nitidus a Variety of Lichen Planus? Archives of Dermatology. 1938;38(4):569.

7. Li AW, Ko CJ, Leventhal JS. Generalized Lichen Nitidus-like Eruption in the Setting of Mogamulizumab and Tremelimumab. Eur J Dermatol. 2017 Jun 1;27(3):325-326. English. doi: 10.1684/ ejd.2017.2989. PMID: 28414193.
8. Daoud M.S., \& Pittelkow M.R. Chapter 27. Lichen Nitidus. Goldsmith L.A., \& Katz S.I., \& Gilchrest B.A., \& Paller A.S., \& Leffell D.J., \& Wolff K, eds. Fitzpatrick's Dermatology in General Medicine, 8e. McGraw-Hill, 2012, pp. 312-316 2018;35(6):866-7.

9. Rallis E, Verros C, Moussatou V, Sambaziotis D, Papadakis P. Generalized Purpuric Lichen Nitidus. Report of a Case and Review of the Literature. Dermatol Online J. 2007 May 1;13(2):5. PMID: 17498424.

10. Jakhar D, Grover C, Kaur I, Sharma S. Dermatoscopic Features of Lichen Nitidus. Pediatric Dermatology.

11. Bilgili SG, Karadag AS, Calka O, Ozdemir S, Kosem M. A Case of Generalized Lichen Nitidus Successfully Treated with Narrow-band Ultraviolet B Treatment. Photodermatol Photoimmunol Photomed. 2013 Aug;29(4):215-7. doi: 10.1111/phpp.12037. PMID: 23815355.

12. Dixit D, Vernekar SS, Giriyan SS. A Clinico-pathological Study of Lichenoid Tissue Reactions/interface Dermatitis. International Journal of Research in Medical Sciences. 2019Apr27;7(4):1002-8.

13. Izak M, Bussel JB. Management of Thrombocytopenia. F1000Prime Rep. 2014 Jun 2;6:45. doi: 10.12703/P6-45. PMID: 24991422; PMCID: PMC4047949.

14. Bettoli V, De Padova MP, Corazza M, Virgili A. Generalized Lichen Nitidus with Oral and Nail Involvement in a Child. Dermatology. 1997;194(4):367-9. doi: 10.1159/000246143. PMID: 9252761.

15. Doğan S, Memis P, Ersoy-Evans S, Gokoz O, Tavil B, Çetin M. Generalized Lichen Nitidus Associated with Neurofibromatosis Type 1 and Juvenile Myelomonocytic Leukemia. Int J Dermatol. 2016 Nov;55(11):e592-e594. doi: 10.1111/ijd.13084. PMID: 26341890.

16. Cho M, Nonomura Y, Kaku Y, Dainichi T, Otsuka A, Kabashima K. Generalized Lichen Nitidus Following Anti-PD-1 Antibody Treatment. JAMA Dermatol. 2018 Mar 1;154(3):367-369. doi: 10.1001/jamadermatol.2017.5670. PMID: 29344609.

17. Rudd ME, Ha T, Schofield OM. An Unusual Variant of Lichen Nitidus. Clin Exp Dermatol. 2003 Jan;28(1):100-2. doi: 10.1046/ j.1365-2230. 2003.01156_8.x. PMID: 12558648.

18. Kubota Y, Kiryu H, Nakayama J. Generalized Lichen Nitidus Successfully Treated with an Antituberculous Agent. Br J Dermatol. 2002 Jun;146(6):1081-3. doi: 10.1046/j.1365-2133.2002.04700.x. PMID: 12072083.

19. Randle HW, Sander HM. Treatment of Generalized Lichen Nitidus with PUVA. Int J Dermatol. 1986 Jun;25(5):330-1. doi: 10.1111/ j.1365-4362.1986.tb02260.x. PMID: 3721671.

20. Taneja N, Mehta N, Arava S, Gupta V. An Unusual Variant of Lichen Nitidus: Generalized Follicular Spinous with Perifollicular Granulomas. J Cutan Pathol. 2020 Sep;47(9):834-9. doi: 10.1111/ cup.13712. Epub 2020 Jul 2. PMID: 32285461.

21. Berman H, Truong A, Cheng CE. Pediatric Generalized Lichen Nitidus Treated with Natural Sunlight Therapy. Pediatr Dermatol. 2019 Sep;36(5):690-2. doi: 10.1111/pde.13915. Epub 2019 Jul 17. PMID: 31313355.

22. Cho-Vega JH, Medeiros LJ, Prieto VG, Vega F. Leukemia Cutis. Am J Clin Pathol. 2008 Jan;129(1):130-42. doi: 10.1309/ WYACYWF6NGM3WBRT. PMID: 18089498.

23. Jin X, Li F, Li X, Zhu W, Mou Y, Huang Y, Zhao H, Gao W, Xia J. Cutaneous Presentation Preceding Acute Monocytic Leukemia: A CARE-compliant Article. Medicine (Baltimore). 2017 Mar;96 (10):e6269. doi: 10.1097/MD.0000000000006269. PMID: 28272239; PMCID: PMC5348187.a 